\title{
Post Liver Transplantation Lympho Proliferative Disorder, Classical Hodgkins Lymphoma Type-A Case Report
}

\author{
Indu R. Nair 1" ${ }^{*}$ Annie Jojo1, Sudhindran Surendran², Dinesh Balakrishnan² \\ ${ }^{1}$ Department of Pathology, Amrita Institute of Medical Sciences, Amrita Viswavidyapeetam, Kochi, India \\ ${ }^{2}$ Department of Surgical Gastroenterology, Amrita Institute of Medical Sciences, Amrita Viswavidyapeetam, \\ Kochi, India \\ Email: "Indurn@aims.amrita.edu
}

Received 18 July 2014; revised 17 August 2014; accepted 15 September 2014

Copyright (C) 2014 by authors and Scientific Research Publishing Inc.

This work is licensed under the Creative Commons Attribution International License (CC BY). http://creativecommons.org/licenses/by/4.0/

(c) (i) Open Access

\begin{abstract}
Post transplantation lymphoproliferative disorder is a lymphoid/plasmacytic proliferation that develops as a consequence of immunosuppression in a recipient of a solid organ/bone marrow/ stem cell allograft. The incidence reported in literature in post liver transplant patients is $1 \%-2 \%$. This case was of a 16-year-old boy who underwent liver transplantation for fulminant hepatic failure and subsequently developed PTLD. Morphologically and immunohistochemically, it was Hodgkin's lymphoma like PTLD, which was the least common type reported in literature.
\end{abstract}

\section{Keywords}

Liver Transplant, Post Transplant Lymphoproliferative Disorder, Classical Hodgkin's Lymphoma Like

\section{Introduction}

Post transplant lymphoproliferative disorder (PTLD) is defined as a lymphoid/plasmacytic proliferation that develops as a consequence of immunosuppression in a recipient of a solid organ/bone marrow/stem cell allograft. The incidence is related to the intensity of immunosuppression, especially following treatment with calcineurin inhibitors. This leads to inactivation of T cells, which, under normal conditions, suppress the viral proliferation. Epstein Barr virus is considered to be the commonest etiological factor and lymphnode is the commonest site affected by PTLD. Most of the cases are seen following heart/lung transplant. The reported incidence is $1 \%$ -

*Corresponding author.

How to cite this paper: Nair, I.R., Jojo, A., Surendran, S. and Balakrishnan, D. (2014) Post Liver Transplantation Lympho Proliferative Disorder, Classical Hodgkins Lymphoma Type-A Case Report. International Journal of Clinical Medicine, 5, $1142-$ 1145. http://dx.doi.org/10.4236/ijcm.2014.518146 
$2 \%$ following liver transplantation.

\section{Clinical History}

This case is of a 16-year-old boy who was flown in to our hospital from the Middle East with features of fulminant hepatic failure. He was investigated for underlying causes, which did not reveal any. All viral markers were negative. An emergency liver transplant was done, from a matched related donor. The explanted liver showed features of submassive necrosis. No abnormal/atypical cell infiltrate was noted. No granulomas were seen.

He developed an episode of subacute rejection, for which he was pulsed with steroids and was then treated with calcineurin inhibitor, tacrolimus. The patient was then assymptomatic. 3 months later, he presented with fever and cervical lymphadenopathy. Routine investigations did not reveal any focus of infection. Hence lymph node biopsy was performed.

\section{Materials and Methods}

Lymph node showed complete effacement of architecture with a polymorphous cell infiltrate (Figure 1). Many large mononuclear/binucleate cells with moderate cytoplasm and large vesicular nuclei with macronucleoli were seen in a background of lymphocytes, eosinophils and histiocytes. Immunohistochemical stains (IHC-DAKO, Germany, prediluted) were done, which showed these large cells to be positive for CD30, EBV-LMP1 and negative for CD45 (LCA), CD15, CD20, CD3 and EMA.

\section{Results}

The morphological and IHC findings were of classical Hodgkin's lymphoma. In the clinical setting of 3 months post transplant, possibility of a post transplant lymphoproliferative disorder was considered. The liver explant was reviewed, and did not show any lymphomatous infiltrate, excluding the possibility of a pre transplant lymphoma (Figure 2).

However, the patient developed features of septicemia and multi organ failure and died within one week. So, a specific treatment could not be given.

\section{Discussion}

PTLDs are a group of heterogeneous lymphoid/plasmacytic proliferation occurring as a consequence of immunosuppression following solid organ transplant.

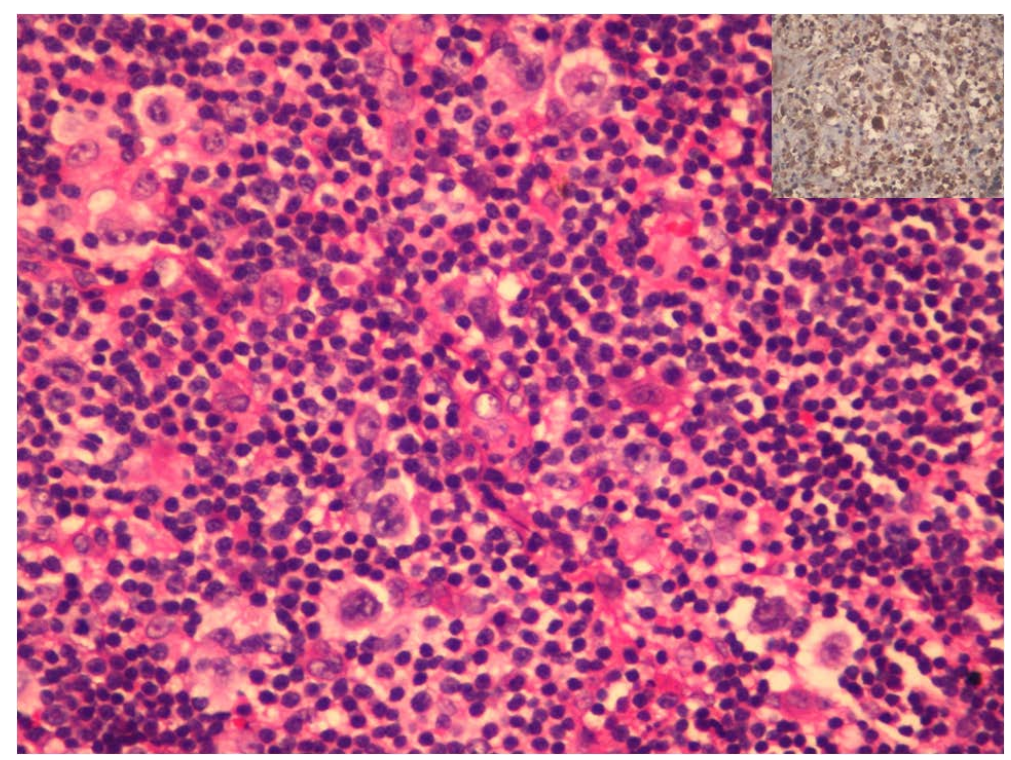

Figure 1. H \& E $\times 400$ showing RS like and Hodgkins like cells. Inset shows EBV-LMP-1 positive large cells. 


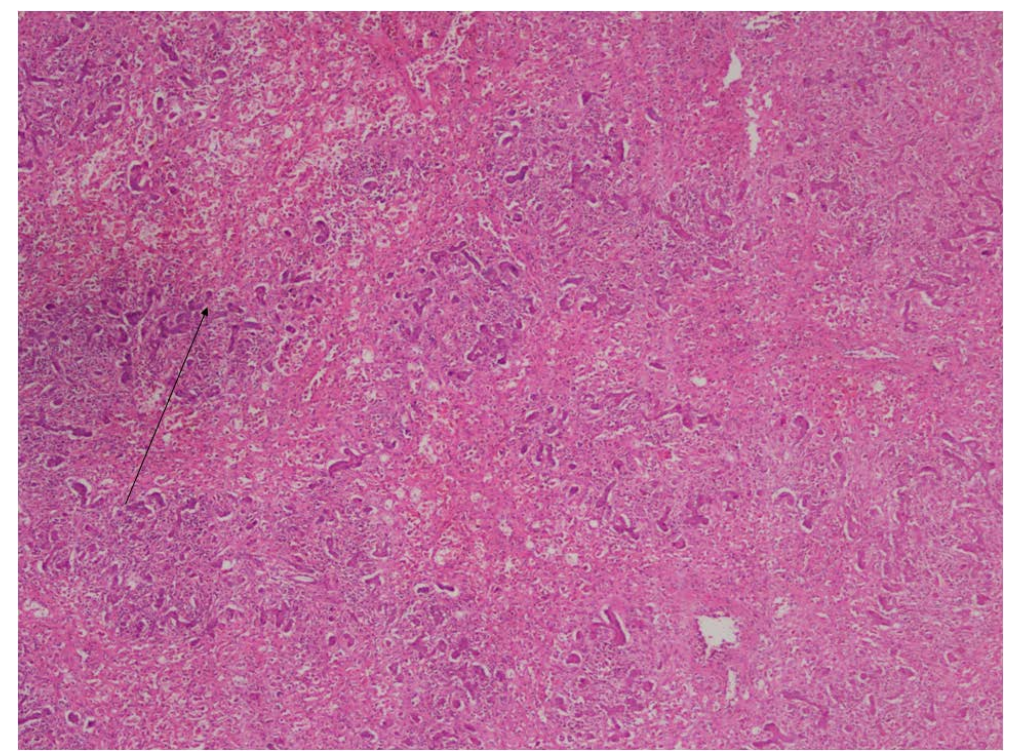

Figure 2. H \& E ×40 showing liver with pan acinar necrosis.

WHO classifies PTLD into the following types:

1) Early lesions,

2) Polymorphic PTLD,

3) Monomorphic PTLD which includes B and T cell neoplasms, and

4) Classical Hodgkin's lymphoma type.

In majority of cases, EBV is found to be the etiological factor. However, in up to $30 \%$ of cases, EBV is not detectable in the proliferating cells. The exact factors involved in these cases are unknown, though other viruses like HHV are implicated [1]. In paediatric transplant recipients, the incidence is much higher. Treatment with immunosuppressive drugs of calcineurin inhibitor type, like tacrolimus and cyclosporine lead to suppression of T lymphocyte function, causing uncontrolled EBV proliferation. The overall incidence of PTLD vary according to the type of organ transplant, with patients receiving renal allografts at the lowest risk, with less than $1 \%$ incidence, hepatic and cardiac allograft recipients with an intermediate risk of $1 \%-2 \%$ and heart-lung/lung/intestinal transplant patients with a high risk of $5 \%$ or greater. Clinical symptoms develop within the first year to as late as 17 years of post transplant [2]. Lymph node is the commonest site affected, followed by GIT, lungs and liver. CNS is the least common site.

In our institute, among the 340 liver transplants done so far, this is the first and only case of PTLD diagnosed. This is a paediatric patient with a pretransplant seronegativity for EBV, and was on tacrolimus. All these factors increased the risk of PTLD.

In adults, monomorphic PTLD is found to be the commonest subtype, whereas in children, the early lesions and polymorphous type are seen more. Among the monomorphic type, Hodgkin's lymphoma subtype is reported to be the least common [1]. The diagnosis is especially difficult because, Reed Sternberg like cells can be seen in early, polymorphic and some monomorphic PTLD. Hence strict criteria are to be followed while making a diagnosis of Hodgkin's like PTLD [3].

In our case, the morphological features resembled that of a polymorphous/classical Hodgkin's lymphoma type PTLD. However, based on the criteria put forth by the WHO [1] and the BCSH (British Committee for Standards in Haematology) and BTS (British Transplantation Society) [3], it was diagnosed as Hodgkin's lymphoma-PTLD. Morphologically, there were large mono/binucleate cells in a background of polymorphous infiltrate. Immunohistochemically, the large neoplastic cells were positive for CD30, EBV-LMP-1 and negative for CD20, CD15, CD3, EMA and CD45 (LCA). Though CD15 negative cases are to be diagnosed with caution, in this case, other markers like CD20 and LCA were negative which ruled out a polymorphous/IMN type PTLD. Such cases are reported in literature [1].

The treatment of PTLD is one of the most controversial areas in solid organ transplantation. The mainstay of treatment include: reduction in immunosuppression, anti B cell monoclonal antibody therapy and chemotherapy. 
The early lesions regress with reduction of immunosuppression, but, at the risk of allograft rejection. Polymororphic and monomorphic lesions might regress or progress. Progressing cases are treated with immunotherapy (anti CD20 antibody) or chemotherapy. In this case, the patient developed features of septicemia and multi organ failure and died within one week, before starting any specific treatment. Infection and multisystem organ failure is a common cause of death in post transplant patients [2].

\section{Conclusion}

This case is presented to highlight the rare complication which can happen in a paediatric patient following liver transplant. Among the PTLDs, Hodgkins lymphoma type is the rarest morphological subtype reported in literature.

\section{References}

[1] International Agency for Research on Cancer (IARC) (2008) WHO Classification of Tumours of Hematopoietic and Lymphoid Tissues. 4th Edition.

[2] Jain, A., Nalesnik, M., et al. (2002) Posttransplant Lymphoproliferative Disorders in Liver Transplantation. A 20-Year Experience. Jorge Reyes Annals of Surgery, 236, 429-437.

[3] Parker, A., Bowles, K., Bradley, J.A., et al. (2010) Diagnosis of Post-Transplant Lymphoproliferative Disorder in Solid Organ Transplant Recipients_-BCSH and BTS Guidelines. British Journal of Haematology, 149, 675-692. 
Scientific Research Publishing (SCIRP) is one of the largest Open Access journal publishers. It is currently publishing more than 200 open access, online, peer-reviewed journals covering a wide range of academic disciplines. SCIRP serves the worldwide academic communities and contributes to the progress and application of science with its publication.

Other selected journals from SCIRP are listed as below. Submit your manuscript to us via either submit@scirp.org or Online Submission Portal.
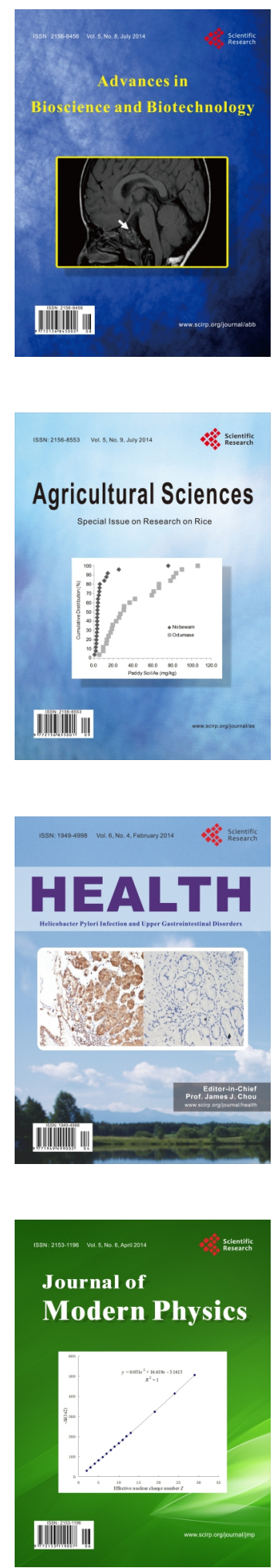
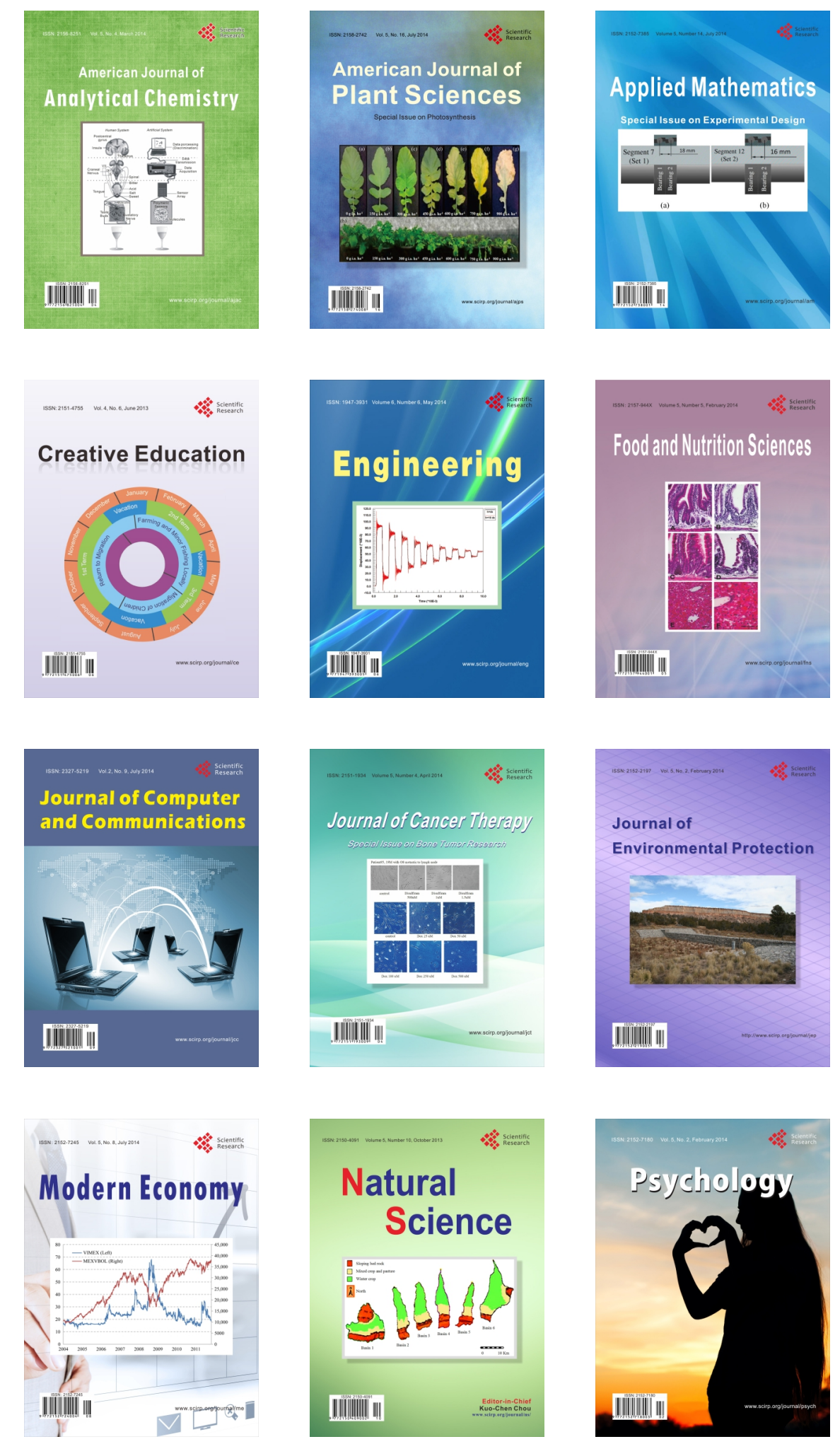